\title{
Twist boundary defects in penta-twinned silver nanowires
}

\author{
Alexander Eggeman, Hu Zhao and Brian Derby \\ University of Manchester, Manchester, England, United Kingdom
}

Networks of metal nanowires are candidates for flexible transparent and conductive films, however the micro- and nanomechanical behaviour of nanowires is still only partially understood. This results partly from a combination of challenging experimental setup, with manipulation of individual nanostructures proving very difficult, hence the relatively limited amount of information on tensile and compressive behaviour of nanowires. There is also a requirement for extremely high spatial resolution when characterizing. Previous studies have shown how spatially resolved diffraction methods (ACOM/SPED /4D-STEM)[1][2] can provide a wealth of information for characterization of complex nanowires.[3]

In this study networks of silver nanowires with the 'penta-twinned' nanostructure [4] were subjected to a range of fatigue cycling to explore both the microscopic change in network structure but also to correlate this with the change in electrical conductivity of the network, which allows the change in network connectivity to be modelled. Furthermore, the networks were produced on holey polycarbonate substrates that could be to allow before and after characterization of the wires and networks.

TEM micrographs of the networks in the as-produced state and after 50000 fatigue cycles are shown in Figure 1a and $\mathrm{b}$. While the wires appear to have remained intact, there are however a number of narrow dark features introduced into the nanowires during the fatigue cycling. The defect density in samples with different fatigue cycles seems to have a monotonic relationship, supporting the idea that these defects are introduced during the mechanical loading of the networks.

SPED analysis of one of these defects is shown in Figure 2. Here unsupervised machine learning decomposition of the 4D data allows the local crystallography of different regions of the wire to be determined. Importantly, the features identified in the previous micrographs are shown to have a different crystal orientation indicating plastic deformation. The general subcrystal orientation being studied is < $819>$ (close to $\langle 101>$ ) as seen in Figures 2c) and 2d) while within the boundary (Figure 2b) there is a considerable signal corresponding to $\langle 115\rangle$. Such a signal can be explained by $\Sigma-3$ boundary, which rotates a $<101>$ oriented subcrystal to $<114>$ and the adjectent (and partially overlapping) $<114$ $>$ subcrystal to $\langle 110\rangle$. However the fault plane is clearly parallel to a $\{110\}$ plane (the wire axis) and not a $\{111\}$, suggesting that the boundary is in fact a twist boundary and not a tilt boundary. Further study of other faults returns misorientations that can only be described by $\Sigma-\mathrm{N}$ twist boundaries. Such a fault suggests a cooperative deformation mechanism with each subcrystal undergoing the same rotational deformation, thus maintaining the symmetry and coherence of the five sub-crystals in the wire, in agreement with similar mechanisms in bending of such wires [3].

The presence of twist boundaries with different coincident site density indicates that while the network is subjected to a macroscopic tensile stress the microscopic stress distribution is far more complex, in particular it is noted in finite element studies of networks that significant shear and torsional stresses are generated particularly when the density of cross-links between ligaments increases [5][6]. This supports the need for greater understanding of the torsional response of nanowires in order to understand the response of nanostructures to real-world loading configurations. 


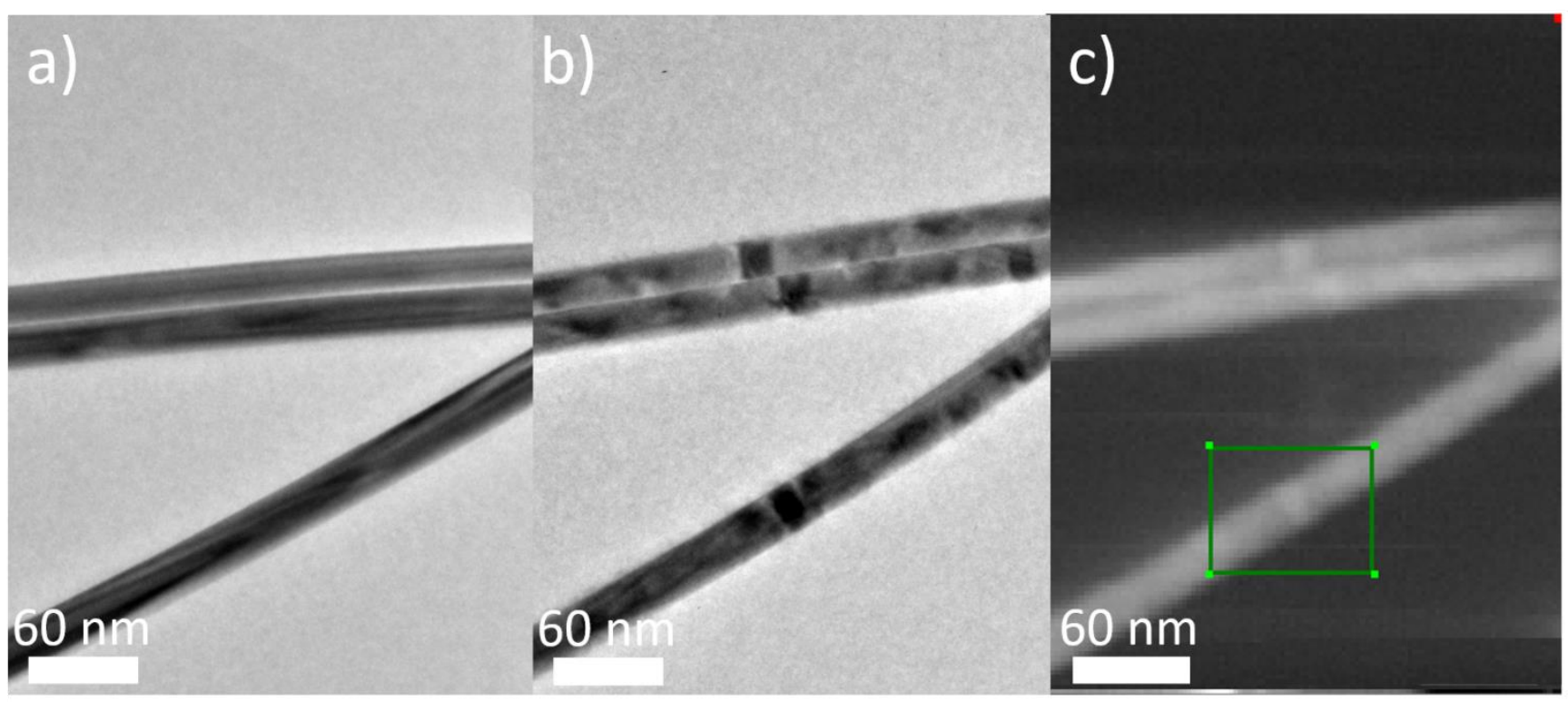

Figure 1. TEM micrographs of penta-twinned silver nanowire network a) before fatiguing. b) after 50000 fatigue cycles. c) virtual bright-field images formed from SPED data of the fatigued sample.
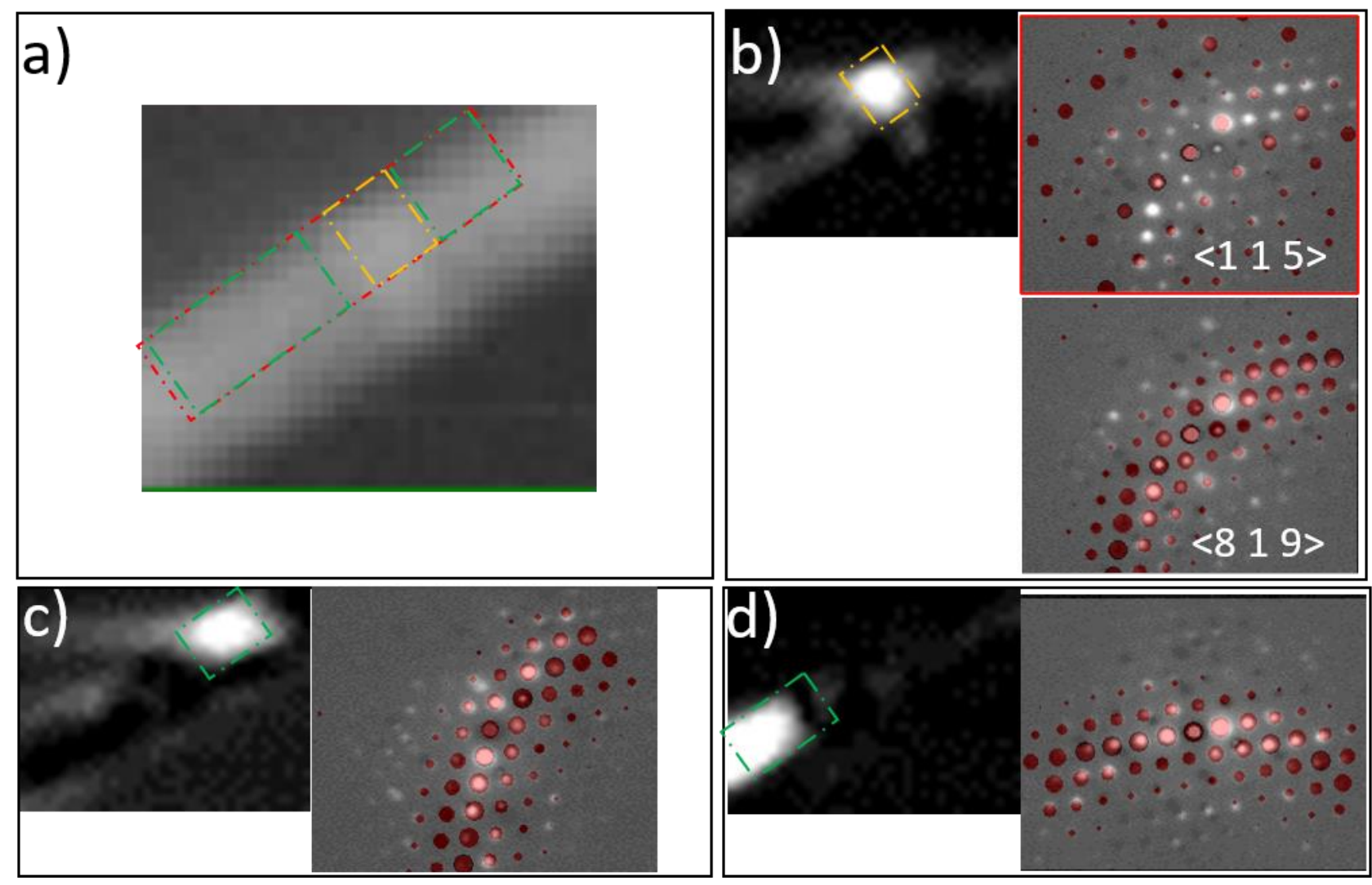

Figure 2. Decomposition of 4D data highlighting the diffraction signals present $b$ ) within the fault regions and c) and d) outside the faulted region.

\section{References}


[1] Rauch, E. F., Véron, M., Portillo, J., Bultreys, D., Maniette, Y. and Nicolopoulos, S. Microscopy and Analysis, 22(6), (2008) S5-S8

[2] Eggeman A. S. Acta Cryst. (2019). B75, p 475-484

[3] Zhao, H, Eggeman, A. S., Race, C. P. and Derby, B, Acta Materialia,185, (2020), p110-118

[4] Sun, M., Cao, R., Xiao, F. and Deng, C. Comput. Mater. Sci. 79, (2013) p289-295.

[5] Bergström, P., Hossain, S. and Uesaka, T. Int. J. Solids Struct. 166 (2019) p68-74

[6] Ranger, A. E and Hopkins, L. F. A new theory of the tensile behavior of paper. Formation and Structure of Paper, Technical Section of the British Paper and Board Makers Association, 1. London (1962), pp. 277-310

[7] The authors acknowledge funding from the Royal Society, the China Scholarship Council and the UK EPSRC through grant number EP/S009493/1. 